Maria Conte

\title{
Osservazioni sulla traduttologia domenicana
}

\section{Un progressivo aumento di controllo sulla circolazione dei saperi}

\begin{abstract}
All'interno del vasto panorama che si spalanca sulla Toscana medievale in merito alla traduzione, l'Ordine domenicano si colloca in primo piano assumendosi la responsabilità della mediazione del sapere. La rigida organizzazione dell'Ordine e la forte influenza che esercita sui mezzi di comunicazione (dalla predicazione all'iconografia) permette ai frati di allestire un progetto solido e unitario di volgarizzamento di testi biblici, patristica, agiografia, letteratura devozionale e di viaggio. Il presente contributo offre una visione d'insieme di tale progetto, ponendo l'accento sulla progressiva presa di coscienza dei domenicani in merito alla loro operazione culturale che dialoga e muta insieme alla società in continuo cambiamento dei comuni toscani.

The Dominican Order plays a fundamental role within the context of medieval translation in Tuscany, because the preachers take the responsibility for the mediation of knowledge. The inflexible organization of the Order and the strong influence it exerts on the media (from preaching to iconography) allow the friars to set up a solid and unitary translation project of biblical texts, patristics, hagiography, devotional, and travel literature. This paper offers an overview of this project, placing the emphasis on the Dominicans' increasing awareness of their cultural operation, which is in constant dialog and evolves within the ever changing society of the Tuscan municipalities.
\end{abstract}

Parole chiave: Dominican Order of preaching, Cultural program, Translation theory

Osservare la traduzione medievale in prospettiva olistica comporta la modifica dell'assunto di una selezione dei volgarizzamenti e della loro funzione in base all'opposizione di genere religioso/classico, e di riconoscere che essi rispondono generalmente a una medesima «grammatica unitaria». ${ }^{1}$ In tal modo si includono

1 A invitare a una visione globale della traduzione medievale sono gli interventi del recente convegno fiorentino confluiti nel volume Tradurre dal latino nel Medioevo italiano. Ma i presupposti

Maria Conte, Università Ca’ Foscari Venezia

Ә Open Access. (c) 2021 Maria Conte, published by De Gruyter. (c) BY This work is licensed under the Creative Commons Attribution 4.0 International License. 
nello scenario nuovi elementi, di tipologia testuale, ambiente di produzione e pubblico che non possono più essere ignorati nello stabilimento di categorie interpretative per i volgarizzamenti: tali variabili influenzano intimamente i singoli risultati letterari ma anche la funzionalità diafasica e diastratica assegnata alla traduzione. Il nostro panorama assume maggiore profondità, dunque, se si considera lo smisurato fenomeno del tradurre nella Toscana medievale primariamente come un fenomeno sociale: gli strumenti della storia culturale consentono di aprire un nuovo osservatorio sull'evoluzione delle teorie del tradurre e di condurre un'indagine trasversale assumendo il punto di vista degli operatori culturali che attuano la trasformazione della società. ${ }^{2}$

Il ruolo chiave dell'Ordine domenicano nella mediazione del sapere è unitamente riconsciuto, e non solo per le traduzioni di ambito religioso; in particolare è stata sottolineata la centralità del convento di Santa Caterina di Pisa, quale crocevia di culture e di figure cardine per lo sviluppo della scrittura volgare. ${ }^{3}$ Nel far fronte alle trasformazioni che modificano profondamente il tessuto sociale dei comuni, i Predicatori estendono il loro modello di controllo culturale anche al campo del volgare, disciplinando la diffusione del sapere

per non ancorarsi esclusivamente al genere letterario e intendere la letteratura religiosa come un prodotto di ambienti ecclesiastici costituente una «traccia di storia» sono già in Delcorno, Produzione e circolazione e in Prosatori minori del Trecento, 1: Scrittori di religione.

2 L'incentivo a procedere su tali indagini giunge dagli studi di Artifoni, Una politica del dittare e Bischetti, Montefusco, Prime osservazioni su "ars dictaminis" orientati sul rapporto tra ars dictaminis, traduzione e egemonia dei saperi.

3 Per un quadro generale sul rapporto tra l'Ordine domenicano e la cultura si vedano almeno Antonelli, L'Ordine domenicano; Bologna, L'Ordine francescano; Bruni, L'apporto dell'ordine domenicano; Delcorno, La lingua dei predicatori; i volumi I Domenicani: storia, figure, istituzioni (1216-2016) e I Domenicani e la letteratura. Specificamente sul convento di Santa Caterina si vedano Banti, La biblioteca del convento di S. Caterina in Pisa; Banti, Libraria nostra communis; il volume Pisa crocevia di uomini, lingue, culture; Pellegrini, I manoscritti dei predicatori. Gli studi più approfonditi sugli attori principali dell’Ordine sono certamente quelli di Emilio Panella e Carlo Delcorno, studi divenuti ormai pietre miliari nel percorso di ricerca sull'Ordine domenicano. Il primo cura un aggiornatissimo blog online (http://www.e-theca.net) attraverso il quale è possibile rinvenire approfondimenti su singoli frati, ma anche su luoghi e conventi dell'Ordine; il secondo ha condotto ricerche che consentono non solo di esplorare nel dettaglio le traduzioni di Domenico Cavalca, il più proficuo tra i volgarizzatori, ma anche di avere un quadro generale della comunicazione in volgare e della sua influenza sulla societas cristiana. In questo senso si veda almeno Delcorno, La predicazione nell'età comunale. Infine, è di immensa utilità per avviare qualunque ricerca in ambito domenicano, il censimento di manoscritti di tutte le opere di frati predicatori, accompagnato da un riassunto delle informazioni biografiche per alcuni di essi: Kaeppeli, Scriptores Ordinis Praedicatorum Medii Aevii. 
con il fine di promuovere l'ortodossia. ${ }^{4} \mathrm{Nel}$ corso di un secolo, elaborano una consapevolezza dell'atto del volgarizzare e acquisiscono il volgarizzamento come strumento comunicativo irrinunciabile per la diffusione del sapere, affiancandolo a quello già rodato della predicazione, affinché possano garantire una risposta adeguata alle esigenze di un pubblico composto prevalentemente da laici. Il presente contributo intende ripercorrere le fasi evolutive della traduttologia che si elabora all'interno dell'Ordine e che comporta acquisizioni in materia di stile, di contenuto da tradurre, di utilità sociale della traduzione, e considera perciò come "volgarizzamenti domenicani” quell'insieme di testi, religiosi e non, fuoriusciti da un ambiente culturale legato all'Ordine dei Predicatori. Saranno tralasciati necessariamente, dunque, i volgarizzamenti di opere latine di autori domenicani realizzati fuori dal controllo diretto dei frati. ${ }^{5}$

\section{Anonimato e dispersione (1260 ca.-1300)}

Nella fase più precoce della traduzione in prosa, orientativamente tra il $1260 \mathrm{e}$ il 1300, le opere rese fruibili al pubblico di illitterati circolano prevalentemente in forma anonima. ${ }^{6}$ L'anonimato contribuisce a rendere meno visibile il limite

4 Sul "controllo ideologico" esercitato dall'Ordine sulla cultura, benché incentrato soprattutto sul latino, si veda Bianchi, Ordini Mendicanti e controllo ideologico.

5 Non saranno dunque prese in considerazione, ad esempio, molte delle versioni della $L e$ genda aurea, per cui si rimanda alla ricerca approfondita di S. Cerullo; e il volgarizzamento della Somme le roi operato da Zucchero Bencivenni, certamente estraneo all'Ordine, ma sulla cui figura si potrebbero aprire nuove indagini, che considerino anche la relazione con gli ambienti religiosi mendicanti. Molti testi della tradizione mistica, come il notissimo Horologium sapientiae sono stati volgarizzati in ambiente monastico ma non domenicano e saranno dunque esclusi dalla nostra analisi. Allo stesso modo dovrà escludersi il volgarizzamento della Summa de casibus di Bartolomeo da San Concordio, attribuito al vallombrosano Giovanni dalle Celle, ma sul quale bisognerebbe aprire un cantiere di lavoro, che sistematizzi innanzi tutto l'immensa tradizione latina, e che individui eventuali redazioni, epitomi, e rimaneggiamenti di quella volgare. Inoltre il contributo non intende trattare i casi di tradizione indiretta di letteratura in volgare che rientrano, ad esempio nella predicazione. Il quadro è riassunto da Cremonini, Non in foglie di parole, ma si vedano anche gli studi essenziali di Carlo Delcorno tra cui si segnala almeno Delcorno, La lingua dei predicatori e Delcorno, Exemplum e letteratura.

6 Escludendo i nomi, eccezionali per molti aspetti, di Bono Giamboni e Brunetto Latini, la condizione di anonimato si verifica anche per la maggior parte della letteratura originale in prosa (si pensi ad esempio al Novellino, alle Sentenze e detti di più filosafi e savi, al Fiore di filosafi e di molti savi imperatori; o alle raccolte di exempla come il Libro dei sette savi, i Conti di antichi cavalieri; i Dodici conti morali). 
tra l'ambiente di realizzazione del volgarizzamento e quello della sua prima circolazione, tuttavia non si può ignorare che testimoni antiquiores, particolarmente autorevoli, di molte tra le prime espressioni della scrittura volgare in italiano si approntano nelle zone di influenza dei conventi domenicani, e attestano perlomeno una immediata ricezione da parte dei frati.

La prima tipologia di volgarizzamenti attribuibile dunque a tale fase aurorale della traduzione domenicana riguarda le Sacre Scritture, nei confronti delle quali è scontato che l'Ordine riservava un certo interesse. Gli autorevoli studi condotti in questo campo ${ }^{7}$ hanno a più riprese dimostrato come - seppure i contorni dei profili di traduttori e copisti siano di difficile definizione - la traduzione della Bibbia non avvenga necessariamente a opera di ecclesiastici, ma sia ascrivibile, anzi, anche a traduttori laici, peraltro spesso di scarsa abilità. ${ }^{8}$ D'altro canto, nel 1242 il capitolo domenicano tenutosi a Bologna, proibisce ai frati la traduzione del libro sacro; ne deduciamo che la pratica doveva essere piuttosto diffusa. Il quadro nebuloso che siamo in grado di ricostruire, lascia quindi emergere «una dinamica di forze contrapposte, tra il desiderio di una lettura 'libera' del testo sacro da parte dei laici e il desiderio di un controllo di tale fenomeno da parte dell'Ordine dei Predicatori». ${ }^{9}$ Tra i libri dell'Antico Testamento riconducibili a una produzione domenicana si può annoverare l'Ecclesiaste, in particolare nella redazione alfa che spicca tra le versioni del libro per le sue felici soluzioni traduttive che rivelano la presenza, sul piano dell'intertestualità, di un immaginario culturale ecclesiastico, e più specificamente vicino a quello della predicazione. La tradizione di questo volgarizzamento, per altro, anche se composta in prevalenza di codici copiati da laici, attraversa $i$ conventi domenicani di Pisa, Siena e Firenze. ${ }^{10}$ Benché non si assicuri con la medesima persuasione il riconoscimento della paternità delle varie versioni dei libri del Nuovo Testamento, d'altra parte sul piano della prima ricezione si distingue tra i più autorevoli testimoni di sillogi neotestamentarie il manoscritto M2 datato alla fine del XIII secolo (il cui antigrafo potrebbe aver conservato la più antica Bibbia completa a noi nota) circolante in ambiente domenicano in

7 Il volume che rinnova l'attenzione sulla questione dei volgarizzamenti biblici è senz'altro $L a$ Bibbia in italiano. A partire da tali ricerche si è allestito anche il catalogo dei manoscritti biblici: Le traduzioni italiane della Bibbia nel Medioevo. Sui singoli libri biblici si sono poi condotte ulteriori indagini che hanno portato a notevoli aggiornamenti: per l'Ecclesiaste disponiamo ormai di un'edizione critica e di uno studio della tradizione molto approfondito (L'Ecclesiaste in volgare); sul Nuovo Testamento si veda Menichetti, Il Nuovo Testamento.

8 Il dato è sotteso agli studi in La Bibbia in italiano.

9 Le traduzioni italiane della Bibbia nel Medioevo, p. XVII.

10 L'Ecclesiaste in volgare. 
epoca piuttosto alta. Il testo conservato in M2 ha una patina linguistica fiorentina, ma giunge molto presto a Ferrara e qui entra probabilmente in un convento domenicano, come si evince da un calendario liturgico aggiunto in chiusura. ${ }^{11}$ Come dimostra il caso di F173, testimone antiquior dei Proverbi, ${ }^{12}$ che si trova conservato a Santa Maria Novella probabilmente da un'epoca antica (fine Duecento o inizio Trecento), la lettura del testo e la sua prima ricezione non bastano a fugare ogni dubbio sull'attribuzione del volgarizzamento. Nonostante il dato della conservazione appaia rilevante, infatti, è difficile che tanto la copia di F173 quanto il volgarizzamento originale siano stati esemplati effettivamente in ambiente conventuale. Secondo Zinelli, la versione italiana dei Proverbi deriva da una versione francese e risulta esemplata per uso certamente laico; il manoscritto lucchese F173, inoltre, è il testimone che più risente dell'influsso linguistico del modello transalpino dimostrandosi fin troppo passivo a livello lessicale, il che allontana l'ipotesi di un traduttore erudito e esperto di testi sacri come un frate domenicano. Per illuminare le restanti zone d'ombra sulla realizzazione dei volgarizzamenti biblici più antichi bisognerà attendere l'avanzamento degli studi filologici sui singoli libri, i quali dovranno necessariamente tenere in considerazione la possibile interazione tra gli ambienti non solo domenicani, ma mendicanti in genere, e la comunità laicale, di cui sono emerse fin qui evidenti tracce sia nella storia della circolazione dei codici, sia nella funzione delle traduzioni. ${ }^{13}$ Altrettanta pazienza si riserverà alla conferma dell'ipotesi della paternità domenicana per la revisione di numerosi libri delle Scritture, revisione che invade il piano stilistico, limando la lingua

11 Il codice M2 è conservato a Venezia presso la BNM, ed è segnato it. I. 2. Già Leonardi, Versioni e revisioni dell'Apocalisse in volgare segnalava il probabile possesso del codice in un convento domenicano probabilmente ferrarese; più recentemente Menichetti, Il Nuovo Testamento ravviva l'ipotesi con nuove suggestioni.

12 Il codice della BNCF segnato Conv. soppr. B. III. 173 è descritto in Pomaro, Censimento dei manoscritti, p. 358. La tradizione dei Proverbi è sistematizzata in Zinelli, «Donde noi metremo lo primo in francescho». Interessante rimarcare due aspetti che emergono dallo studio di $\mathrm{Zi}$ nelli: il codice F173 è l'unico a tramandare esclusivamente i Proverbi ed è il solo che presenta il particolare errore che dà il titolo all'articolo e che dimostra una traduzione pedissequa derivante da un intermediario francese. Inoltre il testo di F173 è l'unico a conservare la sovrabbondante paragrafatura della versione occitana, dovuta alla destinazione orale del testo di partenza.

13 Segnalo un significativo esempio, per quanto tardo, di tale interazione individuabile in due codici quattrocenteschi della tradizione beta dell'Ecclesiaste: il manoscritto V (Città del Vaticano, BAV, Barb. lat. 3931), e il suo collaterale P3 (Paris, BNF, it. 3) sono tra i rari testimoni miniati di libri biblici in italiano e attestano la committenza laicale della realizzazione dei codici (richiesti dalla famiglia Del Balzo) che presentano un'iconografia di impronta chiaramente francescana. 
della traduzione, ma avvicina anche il sensum alla versione della vulgate parisienne, ormai affermatasi con le sue modifiche strutturali, come testo di riferimento nei centri culturali di Parigi e Oxford, da cui si irradia in tutta Europa. La redazione rivista si individua nel ramo D della tradizione dell'Apocalisse, nella versione X3 dei Proverbi, ma potrebbe riferirsi anche ad altri libri. Il rimaneggiamento è da ascrivere, però, a un periodo più avanzato, e sarebbe da collocare, eventualmente, nella seconda fase della nostra scansione, quando la consapevolezza nel governare lo strumento della traduzione sarà senz'altro diversa, e quando Domenico Cavalca avrà già interrotto l'anonimato, traducendo gli Atti degli apostoli, uno dei libri più diffusi e più importanti dal punto di vista dell'edificazione dei fedeli, ${ }^{14}$ e su cui si tornerà nel prossimo paragrafo.

Come noto, un bacino tra i più fertili per la formazione della scrittura prosastica in volgare intorno agli anni '80 del XIII secolo si colloca sull'asse pisano-genovese: l'Ordine domenicano presente nelle due repubbliche marinare potrebbe aver esercitato una supervisione anche su alcune copie realizzate in carcere, conservate in manoscritti di fattura esperta dalle caratteristiche del libro conventuale. ${ }^{15}$ Per alcuni testi possiamo supporre con elementi sufficientemente attendibili che la traduzione si sia approntata in un ambiente domenicano, per quanto in forme primitive e non sistematizzate a livello stilistico.

Il primo intervento che si realizza in tale contesto riguarda un altro genere letterario che rientra appieno tra gli interessi dei frati: l'agiografia. Si tratta della più antica attestazione in volgare di estratti della diffusissima Legenda aurea, raccolta di exempla redatta dal domenicano Iacopo da Varazze. ${ }^{16}$ La silloge non è allestita in modo casuale ma estrapola gli episodi mariani tramandati dall'immensa raccolta fornendo un compendio ben congegnato per l'uso in ambito devozionale. ${ }^{17}$ Il copista prigioniero scrive in un pisano "puro», ${ }^{18}$ ma probabilmente domina anche il francese: il manoscritto che lo tramanda attesta infatti anche un'antologia di miracoli, derivanti tra gli altri dalla stessa Legenda aurea, redatti in una lingua d'oill con tratti pisani. Anche in questo caso,

14 Barbieri, Domenico Cavalca volgarizzatore e Cicchella, «Volendo a pitizione e per devozione». L'edizione è in Domenico Cavalca, Volgarizzamento degli Atti degli apostoli.

15 Sull'influenza dei Domenicani sul carcere genovese si veda Cambi, «In carcere ianuentium». Sui manoscritti realizzati in carcere Cigni, Manuscrits en français, italien et latin; Cigni, I testi della prosa letteraria; Cigni, Copisti prigionieri; si veda, infine, anche il saggio di F. Zinelli in questo stesso volume.

16 Sulla Legenda aurea latina si veda Iacopo da Varazze, Legenda aurea.

17 Il manoscritto che la tramanda è conservato presso la Bibliothèque Municipale di Tours segnato 1008. Cigni, I testi della prosa letteraria, pp. 171-172; Cerullo, La traduzione della Legenda aurea; èdita poi dalla stessa autrice in I volgarizzamenti italiani della Legenda aurea.

18 Cigni, I testi della prosa letteraria, p. 172. 
come in quello dell'Ecclesiaste sono le caratteristiche di qualità della lingua, allestimento antologico e circolazione ecclesiastica che contribuiscono a ipotizzare l'aderenza a un profilo domenicano per il volgarizzatore. Nell'ambito dei volgarizzamenti agiografici rientra anche quello della Storia di Barlaam e Josaphas, un racconto cristianizzato della «meravigliosa conversione» di Buddha, circolante in latino anche in una versione vulgata promossa soprattutto da testi domenicani come lo Speculum Historiale e la Legenda aurea. Tra i numerosi volgarizzamenti di questo intrigante testo agiografico se ne registrano tre, tradotti dal provenzale, su cui sembrerebbe agire una supervisione domenicana. In particolare si inserisce nell'arco cronologico che qui interessa, verso gli anni '80 del Duecento, il testo $\beta$, derivato da un originale lacunoso rispetto al latino, che è tradotto in toscano occidentale e circola sull'asse pisano-genovese: il suo codice più autorevole è copiato all'interno di un atélier che realizza numerosi codici per il convento di San Domenico di Genova, ${ }^{19}$ il che farebbe supporre una partecipazione domenicana anche al progetto della traduzione. ${ }^{20}$ Torneremo anche successivamente su questo testo, interessato da una riscrittura in un periodo cronologicamente più avanzato. Un ultimo esempio, dai caratteri del tutto eccezionali, si allontana sia dalla categoria della prosa che della traduzione verticale: si tratta del poemetto in ottonari rimati a coppie intitolato Quindici segni del giudizio, derivante da un'analoga versione francese. Il testo è tradotto in pisano verso la fine del Duecento, con scarse abilità metriche ma in una lingua libera dall'influenza del testo di partenza, rispetto al quale il traduttore aggiunge una coloritura retorica che, secondo Stefano Carrai, rimanda agli atteggiamenti vivaci della predicazione sia nella gestualità che nel tono. La prima traduzione in rima, conservata in A1, detto "Codice Barbi", sarebbe quindi da ascrivere a un frate domenicano, che dovrebbe rientrare ancora nell'ampio raggio di influenza di Santa Caterina. ${ }^{21}$

Lo statuto pulviscolare della produzione domenicana in volgare nella seconda metà del XIII secolo è espressione di un controllo ancora debole della

19 Sullo scriptorium si veda Gousset, Etude de la décoration filigranée.

20 Il manoscritto citato è il numero 89 della Biblioteca Trivulziana di Milano. L'edizione del testo di $\beta$, basata sul trivulziano, è fornita da Frosini in La Storia di Barlaam e Josaphas secondo il manoscritto 89. La stessa Frosini pubblica anche le edizioni delle altre versioni volgari derivate dal testo provenzale della Storia: la versione G, consultabile in Storia di Barlaam e Josafas. Versione italiana; e la versione $\alpha$ in Storia di Barlaam e Iosafas. Studio sulla tradizione. Si citano anche gli studi che inquadrano l'opera da un punto di vista della storia della produzione e della ricezione: Frosini, Dall'Oriente all'Occidente e Frosini, Il principe e l'eremita.

21 Il manoscritto A1 è conservato presso la BNCF con la segnatura II. VIII. 49. Il testo è studiato in Carrai, Sulla prima traduzione. Per la storia della tradizione francese e italiana si veda anche la scheda di catalogo di Lodone, AnonimQSJD. 
circolazione dei testi; tuttavia, considerando l'intensa attività di studia e scriptoria, emerge l'impellente tentativo di affermarsi come principale veicolo di mediazione culturale nelle zone, come la Toscana, in cui l'Università non è ancora presente. Ciò che riusciamo a discernere chiaramente è il ruolo protagonista del comune pisano nella scena culturale di fine Duecento: il convento di Santa Caterina risponde prontamente al rinnovamento socio-politico delle classi dirigenti e, forte dell'appoggio dell'arcivescovado di Federico Visconti, riesce a inserirsi profondamente in una società divisa a livello sociolinguistico. ${ }^{22}$

\section{Appropriazione dei mezzi comunicativi e sperimentazione (1300-1323)}

A partire dai primi anni del XIV secolo si manifestano i primi risultati del progetto culturale e politico che i frati conducono sul terreno toscano, e in particolare pisano e fiorentino. Al fianco del centro di Santa Caterina, si afferma anche quello di Santa Maria Novella a Firenze, convento che dagli ultimi anni del XIII secolo avvia, sotto la direzione di Remigio de' Girolami, un'intensa attività di produzione libraria all'interno di uno scriptorium che doveva accogliere anche la collaborazione di copisti e miniatori esterni, come dimostrano i manoscritti di elevata fattura, in parte rivisti e annotati dallo stesso Remigio. I codici più preziosi sono quelli di supporto alla liturgia, considerati parte integrante dei paramenti ecclesiastici, mentre gli altri manoscritti tramandano opere latine. ${ }^{23}$ A questa altezza, d'altra parte, si data anche l'ingresso nel convento di alcuni manoscritti di letteratura in volgare, i quali dovevano essere certamente più

\footnotetext{
22 Per un inquadramento della situazione storico-politica a Pisa si veda Poloni, Trasformazioni della società; per il rapporto tra arcivescovado e società comunale è fondamentale Ronzani, Figli del comune o fuoriusciti? Su Federico Visconti basterà segnalare l'edizione del sermonario Les sermons et la visite pastorale.

23 Riporto qui le segnature più importanti che tramandano manoscritti letterari e non liturgici: presso la BNCF si trovano il Conv. soppr. C. IV. 940; il Conv. soppr. E. VII. 938; e il Conv. soppr. G. III. 465 che tramandano opere remigiane, e alla SPK si trova invece il manoscritto qu. 466, con l'Itinerarium di Riccoldo da Montecroce. I miniatori che agivano nello scriptorium su commissione (su cui si veda la voce di Giorgio, Maestri dei Corali) allestiscono invece soprattutto manoscritti liturgici, in particolare i corali del convento, studiati in Orlandi, I libri corali. Per un inquadramento del fervore letterario degli anni remigiani che portarono alla nomina di Studium generale per il convento: Pegoretti, Lo "studium" e la biblioteca. Su Remigio de’ Girolami si veda Panella, Remigio de’ Girolami; Carron, Remigio de’ Girolami.
} 
numerosi di quelli per noi individuabili dalla lettura dell'inventario di Matteo Sardi del $1489 .{ }^{24} \mathrm{Al}$ tempo del priorato remigiano, la comunicazione con i laici rimane ancora prerogativa della predicazione, e solo con il soggiorno a Firenze di Bartolomeo da San Concordio, ${ }^{25}$ in un periodo significativamente complementare all'assenza di Remigio, si verificano le prime esperienze di traduzione presso Santa Maria Novella. Bartolomeo è destinato a restare a lungo una figura di spicco nel panorama domenicano di Pisa, come vedremo nel prossimo paragrafo, ma compie la sua attività traduttoria nel comune fiorentino, volgarizzando un compendio pedagogico allestito da lui stesso anche in latino. Il Libro degli Ammaestramenti degli antichi, auto-traduzione del Liber de documentis antiquorum, stando alle malferme indicazioni della tradizione, dovrebbe essere tradotto tra il 1302 e il 1304, anni cruciali per la politica del comune. ${ }^{26}$ Il Libro raccoglie oltre 1400 sentenze riguardanti ogni aspetto del comportamento morale e ha un discreto successo come opera edificante, giacché è ascrivibile a un genere di immensa fortuna per tutto il XV secolo. Esercitare un controllo sull'universo della morale (non solo attraverso la trattatistica, ma anche tramite la confessione) per l'Ordine dei domenicani costituisce l'apertura di una via preferenziale all'inserimento nelle dinamiche sociali: Bartolomeo riveste la propria figura di una responsabilità pedagogica, istruendo il lettore fedele a un modello di comportamento avallato da lui stesso e promuove il suo ruolo di auctoritas comunicando direttamente con i vertici della società comunale, come attesta la dedica a Geri Spini tramandata da un ramo della tradizione. ${ }^{27}$ L'opera di Bartolomeo seleziona e organizza una tradizione letteraria formata da autori classici e religiosi, fornendo per alcune sentenze la prima traduzione volgare. Le citazioni rimandano così un ca-

24 Probabilmente l'inventario è redatto in occasione dell'ingrandimento della biblioteca avvenuto in seguito alla donazione di libri di Leonardo Dati. Per questo l'inventario registra solo 599 volumi disposti su due file di banchi insieme ad altri 103 conservati in una cassa sub tabulis (Pomaro, Censimento dei manoscritti). Tra i testi elencati troviamo 13 codici che tramandano opere in volgare, quasi tutti allestiti nel XIV secolo, ma per i quali non riusciamo a stabilire la data d'ingresso nella biblioteca. Inoltre nella sezione prestiti si registra un'altra serie di testi volgari, tra cui alcuni già disponibili all'inizio del '300.

$25 \mathrm{Su}$ Bartolomeo da San Concordio si veda Segre, Bartolomeo da San Concordio e lo studio di Vecchio, Quasi armarium scripturarum; mi permetto anche di rimandare a un mio recente lavoro: Conte, Gli Ammaestramenti degli antichi.

26 Inutile ricordare che questo è il periodo in cui si inaspriscono le lotte intestine tra Cerchi e Donati, che costeranno a Dante l'esilio e porteranno i Neri una presa di potere grazie all'appoggio di Bonifacio VIII.

27 La dedica all'esponente di parte nera Geri Spini, banchiere e diplomatico di fiducia di Bonifacio VIII, è in realtà confinata in un ramo basso dell'archetipo, costituito da codici di ambiente 
none gerarchizzato e approvato dall'Ordine. L'operazione di Bartolomeo non si arresta alla letteratura devozionale, ma si apre, nello stesso giro di anni, anche alla storiografia. Il volgarizzamento del De Catilinae coniuratione e del De bello iugurthino sallustiani sono l'unico esempio di traduzione dai classici attribuibile con certezza a un frate predicatore. La scelta di Bartolomeo e la sua contestualizzazione fiorentina non si devono sottovalutare; fino a questo momento, infatti, il genere storiografico è prerogativa di traduttori di tutt'altro profilo, come Bono Giamboni e Brunetto Latini, che intendono attuare un processo per molti aspetti antitetico a quello di Bartolomeo: allargare la possibilità di accedere alla letteratura ai ceti sociali in ascesa, ma illitterati, avvicinando i testi chiave del "repubblicanesimo romano" all'attualità delle lotte intestine comunali. ${ }^{28}$ La disparità di intenti si coglie anche nello stile della traduzione: Bartolomeo mantiene raffinati latinismi del lessico politico, laddove i suoi colleghi laici attualizzano senza riserve, e insiste soprattutto sulla connotazione morale dei personaggi sallustiani inserendo aggiunte nell'aggettivazione. ${ }^{29}$

Il caso di Bartolomeo da San Concordio offre la possibilità di osservare un medesimo traduttore confrontarsi con due operazioni agli antipodi per presupposti e scopi che danno luogo a due risultati differenti dal punto di vista dello stile traduttorio. Gli Ammaestramenti sono un'opera parenetica e moraleggiante, la cui traduzione avviene quasi in simultanea: l'unica differenza tra il testo di partenza e quello di arrivo è il veicolo linguistico. L'autore conduce una traduzione fedele persino all'ordo verborum delle sentenze estratte dai testi, sia perché la struttura testuale costringe a una traduzione per excerpta sia per conservare la veritas del messaggio estratto dalla citazione, come San Girolamo (vera auctoritas nel campo della traduttologia medievale) consiglia di procedere per i versetti della Bibbia, e come in effetti si esegue nel commento delle glosse e nei sermoni. Dal punto di vista lessicale, le innovazioni sono riservate esclusivamente al campo filosofico e morale, mentre nessun latinismo si registra nei campi semantici della politica o dell'economia, scelta funzionale a costruire un immaginario intellegibile per il lettore, che non può in tal modo fraintendere il messaggio edificante e l'invito alla sua applicazione nella contemporaneità.

laico e databili al XV secolo. D’altra parte la coincidenza del soggiorno di Bartolomeo con il periodo di massima notorietà del guelfo nero rendono il dato plausibile, e potrebbe quindi essere stato escluso da una tradizione prevalentemente conventuale dell'opera. I dati sono emersi nel corso dell'allestimento dell'edizione critica, oggetto della mia tesi di dottorato.

28 Sull'argomento sono fondamentali gli studi di Artifoni, Retorica e organizzazione; Artifoni, Gli uomini dell'assemblea; Artifoni, Una politica del "dittare”. Nella stessa ottica anche il lavoro di Tanzini, Albertano e dintorni e Bischetti, Montefusco, Prime osservazioni su "ars dictaminis". 29 Lorenzi Biondi, Le traduzioni di Bartolomeo da San Concordio. 
La coincidenza di autore e traduttore, infine, permette da un lato di controllare meglio la circolazione del testo sotto l'egida domenicana, soprattutto a partire dagli anni '40, e dall'altro rende superfluo il ricorso a dichiarazioni retoriche che giustifichino l'occorrenza della traduzione. ${ }^{30}$ Il genere testuale della storiografia, a cui afferiscono il Catilinario e il Giugurtino, richiede invece una traduzione ad sensum che stimoli la lingua di arrivo a raggiungere lo stile di quella di partenza, assumendo tutte le precauzioni rese necessarie dall'estrema reverenzialità nei confronti del modello:

Onde io, sopra ciò pregato, sì mi brigherò di recarlo in volgare, benché malagevolmente far si possa, per la gravezza del libro, e perché le parole e il modo volgare non rispondono in tutto alla lettera; anzi conviene ispesse fiate d'una parola per lettera dirne più in volgare, e non saranno però così proprie. ${ }^{31}$

La lingua di Bartolomeo è di un registro elevato, ed è ricca di latinismi, soprattutto nel campo semantico della politica. ${ }^{32}$ La tradizione manoscritta dimostra che il volgarizzamento storiografico è sin da subito assimilato dal pubblico laico, appartenente agli strati più alti della nuova borghesia in ascesa; la diffusione è tale che i testi entrano anche in una delle più importanti antologie in volgare che conosciamo oggi, trasmessa dal manoscritto 1538, conservato a Firenze presso la Biblioteca Riccardiana. In questo ambiente di ricezione il nome del frate domenicano svanisce, e nelle rubriche incipitarie troviamo piuttosto messo in risalto quello del committente Nero Cambi, fedele compagno di Geri Spini (il probabile dedicatario degli Ammaestramenti), anch'egli esponente di parte nera entro il 1308.

Forse è anche grazie a questa esperienza che l'Ordine domenicano può collaudare gli strumenti più efficaci per affermarsi nella società e trasferire ai suoi destinatari un universo culturale chiuso e coerente in sé stesso, adattato ai gusti del pubblico laico fiorentino. Bartolomeo da San Concordio esporta un

30 Nel prologo degli Ammaestramenti Bartolomeo si limita a esporre, in modo a dir poco sintetico, la finalità pedagogica e edificante dell'opera: «Sì ccome dice Cassiodoro, lo senno humano, sed egli non è aiutato et restaurato per le cose trovate d'altrui, tosto puote mancare del suo propio. Imperò al savio s'appartiene ched e' non sia contento di suo senno, ma studi diligientemente di cercare l'altrui» e il proprio ruolo di compilator nei confronti del materiale raccolto: «Ma perché la beata sapientia degli antichi inn uno piccolo libro non si potea tutta comprendere, almeno per parte - cioè alquanti loro amaestramenti - avemo curato di raccogliere in questa operetta, secondo il modo della nostra possibilità». Quest'ultimo chiaramente derivato dalla tradizione delle Summae morali del secolo precedente (primo fra tutti lo Speculum maius di Vincenzo di Beauvais).

31 Il testo è riportato secondo la lezione di Basilio Puoti (Il Catilinario ed il Giugurtino).

32 Lorenzi Biondi, Le traduzioni di Bartolomeo da San Concordio. 
modello di traduzione messo a punto nell'ambiente pisano, e si assume in prima persona la responsabilità dell'apertura culturale. Inizia a definirsi dunque la peculiarità domenicana nell'approccio alla scrittura volgare attraverso una notevole attenzione alla qualità della lingua, all'affidabilità dei testi e alla costruzione di un'immagine dell'Ordine precisa e autodeterminata. In tale percorso sperimentale sembrano trovare collocazione tre ulteriori esempi di volgarizzamento di ambiente domenicano per i quali è impossibile determinare una data sicura. Essi sono privi di prologo, di dichiarazioni retoriche sullo stile della traduzione, e non compare il nome dell'autore; tutti e tre, d'altro canto, forniscono una traduzione piuttosto letterale che dimostra una certa fedeltà al testo di partenza. Il primo caso rientra nel filone della letteratura di viaggio, un altro genere testuale di particolare interesse per i Predicatori, sia su un piano interno per formare i frati nell'ottica dell'evangelizzazione della Terrasanta, che su quello esterno per puntare a un coinvolgimento delle confraternite laiche come supporto alla diffusione dell'ortodossia. Si tratta del volgarizzamento pisano del Liber peregrinationis di Riccoldo da Montecroce, frate domenicano che conduce una missione evangelica in Medio Oriente nell'ultimo scorcio del Duecento (1288-1299). Tràdito da un unico codice acefalo e lacunoso, databile all'ultimo quarto del XIV secolo, il volgarizzamento è certamente opera di un traduttore esperto, riconducibile all'ambiente di Santa Caterina. ${ }^{33}$ Il secondo caso ci sposta ancora una volta in zona fiorentina e riguarda un testo devozionale sui generis: il Ludus scaccorum di Iacopo da Cessole, anch'egli domenicano attivo prevalentemente a Genova. L'allegoria della scacchiera per la rappresentazione della società, accompagnata peraltro da un ciclo di illustrazioni molto suggestive e probabilmente autoriali, è infatti innovativa rispetto alla tradizione delle Summae e degli Specula, ed anche per questo il testo ebbe una notevolissima diffusione in ambiente laico e fu tradotto in varie lingue europee. Il volgarizzamento fiorentino, attestato solo in manoscritti tardi, è decisamente più fedele al testo di partenza, a differenza di quello dei rimaneggiamenti allestiti fuori dall'Italia, sia dal punto di vista contenutistico che da quello linguistico: segue il latino in modo pedissequo, «con scostamenti minimi», e per questo l'editore ipotizza una composizione interna all'Ordine domenicano. ${ }^{34}$ Il terzo caso è quello di un aggiornamento della traduzione della Storia di Barlaam e Josaphas derivata da una versione provenzale, ma questa volta la fonte risulta completa e più autorevole rispetto a quella degli altri volgarizzamenti circo-

33 Il codice in questione è segnato II. IV. 53 e è conservato presso la BNCF. L'edizione critica è disponibile nello studio di Il volgarizzamento pisano del Liber peregrinationis.

34 Volgarizzamento del Libellus de moribus hominum. 
lanti, anch'essi, come abbiamo visto, probabilmente realizzati in un contesto di influenza dell'Ordine dei Predicatori. La versione $\alpha$, il cui testimone più antico e autorevole - il riccardiano 1422 - è databile al primo quarto del Trecento e presenta una patina linguistica pisana ascrivibile anche all'originale, è stata probabilmente esemplata nel primo decennio del Trecento. Con questa traduzione, pregevole da un punto di vista linguistico e lessicale, si attesta un rinnovato interesse per la promozione del modello di vita eremitica come esempio per l'edificazione dei fedeli: inizia a delinearsi il pattern tematico per la divulgazione del pensiero domenicano che Domenico Cavalca metterà a punto nei suoi volgarizzamenti e che sarà applicato anche alla predicazione e alla rappresentazione iconografica. A ricollegare la versione all'ambiente di Santa Caterina di Pisa è proprio il manoscritto riccardiano 1422, il cui meditato apparato iconografico dimostra una elevata competenza teorica e una sapiente interrelazione con il testo. ${ }^{35}$ Il risultato librario contribuisce alla promozione di un immaginario di esemplarità della vita ascetica, ma è importante sottolineare che l'eremita Barlaam, con il suo esempio, instrada il principe Iosaphas nel suo percorso di conversione: l'immagine è particolarmente calzante per gli intenti dei frati predicatori, che nel loro apostolato armonizzano la vita contemplativa con l'attività socio-politica proponendosi come guide culturali e spirituali della comunità laicale.

Nell'ambito della patristica, inoltre, sono da segnalare i volgarizzamenti delle Collazioni dei SS. Padri e del De institutis coenobiorum, entrambi probabilmente afferenti all'area pisana dei volgarizzamenti domenicani, ma sui quali non sono disponibili studi sistematici che possano orientare con certezza tali ipotesi. Il primo testo era stato attribuito a Bartolomeo da San Concordio dal suo editore ottocentesco, ma se l'individuazione è forse troppo ambiziosa, si può accettare il profilo generico di un traduttore domenicano, previa un'indagine approfondita e una nuova edizione dell'opera. Per il secondo, invece, l'ipotesi domenicana è avanzata in virtù di una tradizione manoscritta in più casi incrociata con quella delle Vite dei Santi padri di Cavalca e tra cui si rinviene un antico manoscritto pisano-lucchese. ${ }^{36}$

Un rapido affaccio fuori dalla Toscana può dar conto di come il sistema domenicano sia flessibile all'adattamento alle varie realtà in cui agisce e di

35 L'edizione del testo è disponibile in Storia di Barlaam e Iosafas. Studio sulla tradizione. Uno studio sui rapporti della versione con Santa Caterina e sui richiami del testo nell'affresco del Camposanto sono illustrati in Frosini, Testo e immagine.

36 Le informazioni sui due volgarizzamenti sono segnalate in Delcorno, Produzione e circolazione e sono studiate in Chelazzi Dini, Le Collazioni dei santi Padri seguito da Rossi, Le Collazioni dei santi Padri. 
come all'interno di un intricato legame tra ambiente mendicante e societas christiana si realizzino i più interessanti risultati di passaggio linguistico. Il frate Francesco Pipino, attivo tra Padova e Bologna, si dedica - sicuramente entro il 1320 - alla latinizzazione del Devisement dou monde di Marco Polo, basandosi sulla sua versione veneto-emiliana, denominata VA. ${ }^{37}$ La traduzione è significativa soprattutto per le sue conseguenze interne all'ambiente dei Predicatori: la lingua latina ufficializza l'ingresso nel canone letterario domenicano del racconto d'oltremare narrato da un mercante laico contemporaneo; riconoscendolo come autorevole e degno di attendibilità, Pipino incentiva la diffusione del testo e ne autorizza l'uso interno all'Ordine. ${ }^{38}$ Tale trattamento riservato alla figura del mercante veneziano è giustificato se si considera una relazione stretta tra il frate e l'Ordine, di cui si ha notizia certa a partire dal ritorno di Marco Polo a Venezia, ma che potrebbe risalire anche a un periodo più alto. ${ }^{39} \mathrm{Il}$ testo sarebbe poi da mettere a confronto - ma il lavoro è ancora tutto da fare - con l'altra traduzione in latino del Devisement, la versione Z, probabilmente esemplata a Venezia in una collaborazione tra Marco e i domenicani, e che vede soluzioni più severe rispetto ai contenuti della versione originaria. Solo apparentemente inversa alla dinamica di divulgazione del sapere latino in volgare, l'operazione di Pipino è in realtà complementare e ha importanti implicazioni nell'esercizio domenicano di controllo di un sapere selezionato, che sia esso religioso o laico, antico o contemporaneo.

Nel corso dei primi anni del XIV secolo si assiste, dunque, al definirsi di una serie di prerogative della traduzione domenicana dal punto di vista tematico e stilistico, che mirano uniformemente all'appropriazione di un canale di distribuzione del sapere. In questi anni prende forma un programma di riadattamento linguistico di una letteratura selezionata all'interno della cultura domenicana in cui si vede il riflesso di radicale mutamento della cir-

37 Sul frate Francesco Pipino si veda Zabbia, Pipino, Francesco. La composizione della latinizzazione è datata tra il 1310 e il 1322 da Gadrat-Ouerfelli, Lire Marco Polo au moyen age, pp. 67-68. L'opera, intitolata Milion, è edita in Mirka Pavlova $z$ Benàtek e in Liber domini Marchi Pauli de Veneciis, ma sono ancora molte le questioni rimaste in ombra sulla tradizione del testo.

38 Il prologo aggiunto dal frate si apre con parole di riconoscimento nei confronti del contenuto e dell'autore del testo: «Librum prudentis honorabilis ac fidelis viri domini Marchi Pauli de Venetiis».

39 Marcello Bolognari ha recentemente comprovato i sospetti di tale rapporto con il ritrovamento di un documento che attesta la presenza di Marco come testimone per l'accettazione di un lascito da parte di Giovanni dalle Boccole al convento di San Giovanni e Paolo, ruolo che l'Ordine riservava solo a persone di fiducia (per l'edizione e l'analisi storico-culturale del documento si veda Bolognari, Marco Polo e il convento dei SS. Giovanni e Paolo. 
colazione del pensiero. L'Ordine domenicano affida la responsabilità di tale circolazione a operatori linguisticamente competenti per differenziare diastraticamente contenuti e destinatari del sapere; come vedremo, l'esperienza di Bartolomeo da San Concordio diverrà paradigmatica nel rappresentare il bilinguismo del mediatore culturale domenicano.

\section{Affermazione del ruolo di auctoritas e promozione di un'identità (1323-1357)}

Il 1323 può essere considerato un anno cruciale, un vero momento di svolta per la diffusione del pensiero domenicano: è l'anno della canonizzazione di Tommaso d'Aquino, dalla quale l'Ordine incamera una nuova vitalità che consente un deciso rafforzamento. L'evento segna la riuscita dell'intensa campagna di divulgazione della teologia tomistica messa in atto dai domenicani a partire dal 1277 (anno in cui il vescovo Tempier metteva all'indice 15 tesi risalenti all'aquinate) favorita anche da alcune dinamiche socio-politiche e ecclesiastiche, prima fra tutte la diffusione delle eresie francescane e la condanna da parte di Clemente V, nel 1312 durante il Concilio di Vienne, di tre teorie del francescano spirituale Pietro di Giovanni Olivi. La condanna riabilita implicitamente la visione aristotelico-tomistica e può essere infatti resa definitiva nel 1327, dal papa Giovanni XXII, quando le teorie del filosofo aquinate sono ormai considerate ufficialmente ortodosse. A partire dal 1323, insomma, assistiamo a un notevole incremento degli investimenti sulla costruzione dell'identità dell'Ordine domenicano che trova nella figura di Tommaso un volto rappresentativo, più di quanto non sia stato quello dello stesso fondatore: il filosofo è al centro di numerosi cicli iconografici attraverso i quali si promuove l'immagine di un magister responsabile della mediazione della Parola e della cultura nei confronti della societas christiana; è questa la forma estrinseca che l'Ordine vuole conferire alla percezione di sé presso i laici. ${ }^{40}$ La proliferazione di interventi artistici e architettonici in questo periodo è possibile ancora una volta in virtù del solido ruolo di pilastro sociale che i domenicani sono riusciti a costruirsi all'interno delle città. A Pisa il supporto politico è garantito dal susseguirsi di arcivescovadi domenicani, il primo di Oddone della Sala (1312-1317) e il secondo di Simone Saltarelli (1323-1328 e 1330-1342); entrambi fanno fronte abilmente alle crisi politiche innescate dal complesso rapporto con le figure imperiali in-

40 Cannon, Religious poverty, visual riches. 
staurando un profondo legame con alcune famiglie illustri, legame che mantiene l'equilibrio dei rapporti con il papato. ${ }^{41}$ Attraverso un'intensa attività di predicazione e tramite il vincolo sacramentale della confessione i domenicani consolidano i rapporti con laici eminenti e facoltosi, i quali destinano ingenti donazioni ai conventi. In questo periodo Santa Caterina, sotto la direzione artistica di Iacopo Donati, Domenico Cavalca, Bartolomeo da San Concordio e Giordano da Pisa può ristrutturare la facciata (1327), affrescare il Camposanto (1335), rinnovare la biblioteca (1338), commissionare più di un ciclo iconografico (1340 e 1345). ${ }^{42}$ Presso Santa Maria Novella, invece, agisce Iacopo Passavanti, figura poliedrica che risponde perfettamente al modello di intellettuale diplomatico caratteristico dei più incliti esponenti dell'Ordine: egli possiede legami di sangue con una delle famiglie più illustri della città, i Tornaquinci, e coltiva relazioni strette con i vescovi fiorentini. ${ }^{43}$ Sotto il suo controllo si avviano importanti rinnovamenti strutturali del convento e iniziative sulla stessa linea di quelle pisane, come la fondazione della biblioteca (1338), la committenza di importanti cicli pittorici (1336-8) e l'ideazione del Cappellone degli Spagnuoli (realizzato successivamente, nel 1365).

41 Nel ristabilire un rapporto pacifico col papato dopo l'ingresso di Ludovico il Bavaro e Niccolò V a Pisa, un ruolo di mediazione è certamente da attribuire a Fazio Novello, il quale ebbe una stretta relazione con i domenicani e in particolare con Bartolomeo da San Concordio, come si deduce dai lasciti nel suo testamento. Per approfondire la situazione storico-politica si vedano gli studi di Ronzani: Ronzani, La chiesa pisana al tempo di Enrico VII; Ronzani, Saltarelli, Simone; Ronzani, Figli del comune.

42 La facciata di Santa Caterina, la più antica in marmo bianco, è finanziata dalle donazioni della famiglia Gualandi intorno al 1327. Il Camposanto è affrescato da Buonamico da Buffalmacco intorno al 1335, e è ormai accertato che Domenico e Bartolomeo abbiano contribuito all'ideazione concettuale dell'affresco (Frugoni, Altri luoghi cercando il paradiso; Bolzoni, La rete delle immagini; il volume Atlante delle Tebaidi e dei temi figurativi). Tanto è vero che nelle iscrizioni multilingui dei cartigli sono leggibili rimandi alla predicazione di Giordano da Pisa e ai temi morali affrontati da Domenico nei suoi trattati. Di poco successiva è anche la pala d'altare con il Trionfo di San Tommaso, dipinta da Lippo Memmi nel 1340 in cui il frate è rappresentato in apoteosi, con un libro in mano, a simboleggiare la trasmissione del sapere verso la comunità di chierici e laici rappresentata ai suoi piedi. Questo, insieme ad altre modifiche del convento, è finanziato dal lascito del giudice di diritto canonico Bonagiunta da Calcinaia del 7 marzo 1335 (il testamento è conservato presso l'Archivio Diplomatico di Pisa nel fondo conv. di S. Cat., segnato conv. n. 91). Infine è del 1345 il Polittico di San Domenico realizzato da Francesco Traini su commissione di Albizzo delle Stadere, il quale cita Bartolomeo da San Concordio nel suo testamento.

43 Aggiornamenti fondamentali sulla biografia e le opere di Iacopo si trovano nelle ricerche di Agnese Macchiarelli: Macchiarelli, Per la biografia; Macchiarelli, Iacopo Passavanti e la Theosophia. 
La diffusione di testi in volgare è parte integrante del fervore culturale che si accende in questi anni tra i frati domenicani ed è alimentata sia dalla stesura di nuovi volgarizzamenti, che dalla produzione libraria. Gli operatori culturali domenicani controllano tutto il processo, dall'ideazione alla sua manifestazione, consci dell'importanza di tutte le fasi per arrivare al pubblico in modo determinante. Scrittore instancabile e protagonista carismatico di questo periodo è senz'altro Domenico Cavalca, il quale incrementa sensibilmente la propria produzione letteraria intorno agli anni Trenta, dopo essersi dedicato alla predicazione e all'organizzazione pedagogica di conventi femminili. Domenico condivide gli anni della fioritura del convento pisano con Bartolomeo da San Concordio ma, a differenza del suo confratello, il suo apostolato si svolge dall'inizio nella città natale e si esprime primariamente nel campo dell'educazione devozionale in volgare; mentre Bartolomeo in questi anni si sposta piuttosto nel campo - complementare - della pedagogia interna al convento e si dedica alla compilazione della Summa de casibus conscientiae per istruire i frati all'aggiornata pratica della confessione. La scelta cavalchiana delle opere da tradurre riflette gli interessi preponderanti dell'Ordine individuati sin qui e definisce in modo più determinato una letteratura religiosa in volgare che può a ragione definirsi domenicana: la patristica edificante del Dialogo di San Gregorio e delle lettere di San Girolamo, l'agiografia delle Vitae patrum, le Sacre Scritture degli Atti degli apostoli. Estremamente significativo, inoltre, che l'ultima opera di Cavalca, l'Esposizione sopra il simbolo degli apostoli, sia costituita in gran parte da un volgarizzamento, seppur non esplicitato, del De articulis fidei di Tommaso d'Aquino; a sottolineare ancora quanto sia influente il pensiero del filosofo, che finalmente negli anni Quaranta può esser diffuso anche in volgare. Le dichiarazioni proemiali, per quanto rispondano ai topoi retorici ormai standardizzati nella tradizione, ci informano sull'avanzamento della riflessione domenicana sulla traduttologia. Nelle scelte traduttorie cavalchiane vediamo concretizzarsi su un piano concettuale ciò che Bartolomeo aveva impostato a livello pragmatico: al di là delle rivendicazioni di utilità per giustificare l'occorrenza del volgarizzamento emergono osservazioni sullo stile della traduzione che sembrerebbe privilegiare il sensum del testo, piuttosto che la lettera.

Ma volendo incominciare, trovai il suo Latino in tal modo dettato per grammatica, che non mi pare di poter seguitare al tutto l'ordine della lettera; perciocché tal cosa par ben detta per grammatica, che ridotta in volgare secondo l'ordine della parole pare niente, e meno utile. ${ }^{44}$

44 Il testo citato segue la lezione di Domenico Cavalca, Volgarizzamento del Dialogo di San Gregorio. 
Per analizzare tali scelte e le sue effettive applicazioni nella traduzione disponiamo di autorevoli e aggiornati studi, i quali dimostrano in realtà che lo stile traduttorio di Cavalca è uniforme a quello di Bartolomeo da San Concordio: oscilla in maniera ponderata tra soluzioni ad sensum e ad verbum della forma latina. ${ }^{45}$ La traduzione letterale nel Dialogo di San Gregorio è perseguita soprattutto in materia di fede, e rende il testo di partenza in modo chiaro e esplicito, al fine di evitare interpretazioni eterodosse. Tale oscillazione si riscontra anche nelle Vite dei santi padri e, nonostante le variabili interpretative imposte dalla presenza di un atélier di traduzione, credo sia significativo che la letteralità sia riservata ai Verba seniorum, la parte della raccolta che più si avvicina al genere sentenziale; invece negli exempla si procede a una traduzione interpretativa, raggiungendo il massimo grado di libertà per la Vita Antonii, tra le più note della raccolta. Mettendo a sistema le informazioni di queste approfondite indagini, risulta coerente anche la recente analisi sull'Esposizione in cui si nota che Cavalca è decisamente più fedele alla littera del De articulis fidei di Tommaso di quanto non lo sia Giacomo della Lana, nel suo volgarizzamento dello stesso testo, inserito nel commento dantesco. Le sentenze tomistiche, dunque, sono trattate come materiale particolarmente autorevole, e devono essere tradotte in modo schietto, asciutto, e all'occorrenza letterale, con la stessa attenzione che si dedica alle parole dei magistri e ai libri sacri. Coerentemente con gli insegnamenti di una formazione scolastica, Cavalca intende separare nettamente la traduzione dall'esegesi, attitudine che non troviamo invece nei volgarizzamenti di ambiente laico, decisamente più inclini alla glossa intertestuale e alla traduzione interpretativa.

Gli effetti delle riflessioni pisane giungono fino a Firenze e si assiste al definitivo allineamento della politica dei due conventi, che avevano avviato i primi scambi all'inzio del secolo attraverso la figura di Bartolomeo. A partire dalla fine degli anni Trenta possiamo individuare un contatto concreto tra le due città nella presenza a Pisa di almeno due personaggi fiorentini: il primo è Simone Saltarelli, già priore a Santa Maria Novella nel 1300, che in qualità di vescovo a Pisa intensifica i rapporti tra le due città attraverso donazioni di libri e suppellettili; ${ }^{46}$ il secondo è Iacopo Passavanti, il quale si trova a Pisa probabilmente in qualità di lettore, intorno al 1335 per poi tornare a Santa Maria Novella e divenire il principale promotore della diffusione del pensiero domenicano nel comune. La principale espressione di tale propagazione riguarda direttamente

45 Sul Dialogo si veda Verlato, «Sforzandomi di seguitare le parole»; sulle Vite Domenico Cavalca, Vite dei Santi Padri; sugli Actus: Barbieri, Domenico Cavalca volgarizzatore; sul De articulis fidei Volpi, «Diremmo come li pone Fra' Thomaxe de Aquino».

46 Ronzani, Saltarelli, Simone. 
la produzione libraria. Da un lato il convento di Santa Maria Novella rinnova la propria biblioteca, ${ }^{47}$ dall'altro credo che sia riconducibile all'influenza del pensiero domenicano anche l'incremento della diffusione di testi agiografici e devozionali. Intorno agli anni Trenta-Quaranta infatti prendono avvio le tradizioni fiorentine delle Vite dei Santi Padri e degli Ammaestramenti degli antichi. ${ }^{48}$ Passavanti si dimostra ben consapevole dell'importanza dell'affidabilità dei testi, e che tale autorevolezza deve essere avallata da una personalità precisa di operatore culturale, che per la prima volta viene programmaticamente delineata. Riusciamo facilmente a immaginare che la supervisione e il controllo che i domenicani esercitano sui testi latini, ricontrollandoli e revisionandoli, si estenda a quest'altezza anche ai testi volgari:

In certi libri della Scrittura e de' dottori che sono volgarizzati si puote leggere, ma con buona cautela, imperò che si truovano molto falsi e corrotti, e per difetto degli scrittori, che non sono comunemente bene intendenti, e per difetto de' volgarizzatori, i quali i passi forti della santa Scrittura e' detti de' santi sottili e oscuri non intendendo, no gli ispongono secondo l'intimo e spirituale intendimento, ma solamente la scorza di fuori della lettera, secondo la gramatica, recano in volgare (. . .) E così ogni uomo se ne fa esponitore, con ciò sia cosa che, a volerla bene volgarizzare, converrebbe che l'autore fosse molto sofficiente, che non pur gramatica, ma egli converrebbe sapere ben teologia e

47 Intorno al 1338, come si è già accennato, la fondazione del primo nucleo della Libraria è riconducibile all'azione di Passavanti in qualità di operaius del convento.

48 I tre manoscritti antiquiores della tradizione degli Ammaestramenti sono allestiti nel $1342 \mathrm{e}$ nel 1343 e decorati da due miniatori di scuola fiorentina con un ciclo iconografico molto simile, si tratta dei codici II. II. 319 e Pal. 600 della BNCF e del Castiglioni 3 della BNB. Per una descrizione accennata dei codici e per una loro provvisoria sistemazione devo rimandare nuovamente al mio contributo Conte, Gli Ammaestramenti degli antichi. Il Vitt. Em. 1189 della BNCR tramanda invece l'antiquior della Vulgata delle Vite e è allestito nella notissima bottega di Francesco da Ser Nardo da Barberino. Una descrizione in Bertelli, I codici di Francesco. Per le Vite si registra una vera e propria vulgata fiorentina, separata dalla versione pisana del testo, più vicina all'originale. Per gli Ammaestramenti la situazione è diversa giacché il testo ha probabilmente una sua forma compiuta nell'ambiente fiorentino. In entrambi i casi, d'altra parte, i manoscritti realizzati verso gli anni ' 40 a Firenze sono testimoni di elevata fattura e di particolare attendibilità. L'iconografia trasmessa in questi oggetti risente senz'altro delle altre manifestazioni artistiche realizzate all'interno dell'Ordine in questo periodo. Confrontando queste testimonianze con altre fabbricazioni librarie dello stesso tipo realizzate fuori dall'Italia si potrebbe iniziare a pensare a un programma di supervisione testuale e artistica sulla letteratura volgare. Ad esempio, oltre ai già citati, si prenda in considerazione il codice Chig. M. VIII. 158, che tramanda l'unica attestazione del volgarizzamento veneziano del De Regno di Tommaso d'Aquino concluso Tolomeo da Lucca, realizzato poco dopo la morte dello stesso Tolomeo, vescovo di Torcello: il codice rappresenta un vero e proprio Trionfo di San Tommaso in trono nella carta iniziale e ripetute raffigurazioni di Tolomeo come magister e mediatore di contenuti filosofici. 
delle Scritture sante avere esperta notizia, e essere rettorico e essercitato nel parlare volgare, e avere sentimento di Dio e spirito di santa devozione, altrimenti molti difetti vi si commettono, e sono già commessi. ${ }^{49}$

In questo citatissimo passo si esprime la consapevolezza raggiunta dalla traduttologia domenicana: si ricerca nella traduzione una espressione del sensum senza tralasciare il carico semantico del verbum..$^{50}$ La profonda cognizione autoriale di Passavanti si manifesta nella scelta di allestire un'opera non soltanto bilingue, come era il Liber di Bartolomeo, ma bifronte: tra lo Specchio della vera penitenzia e la Theosophia la separazione diastratica evidente nella scelta linguistica invade anche il piano contenutistico. Se, ad esempio, nel trattato indirizzato agli illitterati i riferimenti patristici sono costantemente accostati all'esegesi per indirizzare il comportamento devozionale, quando si rivolge a un pubblico di "addetti ai lavori” Passavanti può lasciare i rimandi irrelati. $^{51}$ Attraverso una decisa affermazione autoriale, sconosciuta ai suoi confratelli, Passavanti esplicita nel prologo l'ontologia di un'operazione culturale tutta domenicana:

Io, frate Iacopo Passavanti, dell'Ordine de' Frati Predicatori minimo, pensai di comporre e ordinare certo e spezial trattato della penitenzia. E a ciò mi mosse il zelo della salute dell'anime, alla quale professione dell'Ordine mio spezialmente ordina i suoi frati, provocommi l'affettuoso priego di molte persone spirituali e devote, che mi pregarono che quelle cose della vera penitenza che io per molti anni e spezialmente nella passata quaresima dell'anno presente milletrecentocinquantaquattro, avea volgarmente al popolo predicato, ad utilitade e consolazione loro e di coloro che le vorranno leggere le riducesse a certo ordine per iscrittura volgare, sì come nella nostra fiorentina lingua volgarmente l'avea predicate. Onde, non volendo né dobbiendo negare quello che la carità fruttuosamente e debitamente domanda, porgo la mano collo ingegno a scrivere e per volgare, come fu principalmente chiesto, per coloro che non sono litterati, e in latino per li chierici, a' quali potrà esser utile e per loro e per coloro i quali egli hanno amaestrare o predicando, o consigliando, o le confessioni udendo (. . .). ${ }^{52}$

Per dar conto di come l'onda lunga delle acquisizioni passavantiane manifesta i suoi effetti, meritano una menzione anche le generazioni successive di frati domenicani, per quanto rientrino nello scorcio conclusivo dell'arco cronologico

49 Il testo è citato secondo l'edizione critica Iacopo Passavanti, Lo specchio della vera penitenzia.

50 Così anche Verlato, «Sforzandomi di seguitare le parole», p. 207.

51 Uno studio approfondito delle fonti, particolarmente rivelatore per il riuso diffuso di testi tomistici, è quello che sta conducendo Agnese Macchiarelli, che ringrazio per avermi fornito in anteprima le pagine del suo contributo in corso di stampa: Macchiarelli, Iacopo Passavanti e la Theosophia.

52 Iacopo Passavanti, Lo specchio della vera penitenzia, Prol. 25-27, p. 212. 
che qui interessa. I domenicani attivi tra il 1360 e il 1420 non rinunciano allo strumento del volgare, sia in traduzione che in forma di letteratura originale: $\mathrm{i}$ loro interessi si mantengono ben saldi soprattutto sulle linee scritturali, agiografiche e morali e si indirizzano principalmente a un pubblico di devoti, vicini all'Ordine per l'afferenza a gruppi confraternali, e a un pubblico femminile, costituito dalle consorelle domenicane e dalle terziarie anch'esse vicine all'influenza dei conventi. ${ }^{53} \mathrm{Nel}$ panorama toscano primeggia ormai incotrastato il convento di Santa Maria Novella, infatti Santa Caterina subisce un grave spopolamento durante la peste del 1348 e non recupererà più l'antico splendore: nella seconda metà del Trecento i frati si occupano di conservare il ricordo delle origini auree del convento attraverso la stesura della Chronica da parte di Domenico da Peccioli, che erige un vero e proprio monumento alle figure che lo hanno animato, prima fra tutte Bartolomeo da San Concordio, di cui il cronista fu allievo, e che lo incaricò della scrittura. ${ }^{54}$ A Santa Maria Novella riconosciamo invece l'attività di frati particolarmente vicini all'immaginario umanistico, che contribuiscono all'allargamento della biblioteca del convento, primo fra tutti Leonardo Dati (1360 ca.-1425), a cui è attribuito un poemetto in ottave di argomento astronomico intitolato La sfera. ${ }^{55}$ Di formazione fiorentina anche l'illustre Giovanni Dominici, accorato promotore di interpretazioni mistiche e spirituali del testo sacro, svolse un apostolato tormentato tra Venezia e Firenze, durante il quale si dedicò principalmente alla predicazione e alla pedagogia: a lui si attribuisce il volgarizzamento della Vita Mariae Oignacensis, ${ }^{56} \mathrm{ma}$ anche trattati di argomento devozionale, tra cui il più importante è senz'altro il Libro d'amor di carità. Inserito nel dibattito culturale del suo tempo, si scagliò con veemenza contro Coluccio Salutati e la sua interpretazione "pagana" dei classici, senza rifiutare d'altro canto un umanesimo coerente con il «bene

53 Sul tema si veda anche Delcorno, Produzione e circolazione, p.13. Per vedere nuovamente integrata anche la traduzione dei classici bisogna attendere la metà del XV secolo con i volgarizzamenti di Seneca e Cicerone esemplati da Filippo da Strada su cui Stampa meretrix; Dell'Oso, Un domenicano contro la stampa.

54 Vecchio, Quasi armarium scripturarum. La Chronica è stata pubblicata integralmente da Bonaini (tomo II, vol. 2) come allegato al commento delle Istorie pisane di Roncioni. Bonaini fornisce il testo della cronaca di Pisa accanto a quello di altri conventi e commenta alcuni passaggi precisando soprattutto la datazione degli eventi citati. L'edizione critica del solo prologo si trova in Panella, Cronica del convento di Santa Caterina.

55 Si veda Bertolini, Censimento dei manoscritti della Sfera del Dati; Bertolini, L'attribuzione della Sfera del Dati; Segatto, Un'immagine quattrocentesca del mondo.

56 Uno studio in prospettiva di un'edizione critica è quello di Vanelli Coralli, Il volgarizzamento della Vita Mariae Oigniacensis. 
supremo della salvezza». ${ }^{57}$ Altra figura centrale e esemplare per gli interessi dell'Ordine nella seconda metà del secolo è Tommaso Caffarini, sicuramente vicino anche al Dominici, con cui spartì l'ammirazione per Caterina da Siena: Caffarini fu il primo seguace della santa, suo confessore e biografo. La sua attività si estende da Siena a Venezia e si fonda sull'intimo legame tra predicazione e meditazione: Caffarini invita alla lettura delle vite dei santi, e soprattutto delle sante, di cui si dedica a volgarizzare le Vite per l'edificazione delle sue consorelle domenicane. $^{58}$

Nello stretto legame tra predicazione e pedagogia, i Predicatori continuano a imitare il modello di intellettuale incarnato da Domenico Cavalca, che propone la composizione volgare e la traduzione del sapere come muta predicatio, per accompagnare l'edificazione del fedele anche nella sua quotidianità e trasferendo contemporaneamente un pensiero conforme a quello dell'Ordine.

\section{Conclusioni}

L'Ordine domenicano, in conclusione, promuove un tipo di mediazione del sapere che orienta la diffusione su un'asse verticale, alla cui sommità posiziona la personalità del magister, intellettuale duttile e accorto che abbiamo visto incarnato in alcune figure-chiave quali Bartolomeo da San Concordio, Domenico Cavalca e Iacopo Passavanti. Attorno a questi tre personaggi si elabora una vera e propria traduttologia che interpreta il volgarizzamento come veicolo di selezione di un sapere approvato dall'Ordine. Fondamentale, dunque, risulta anche controllare la trasmissione dei testi selezionati; infatti la circolazione dei libri collabora alla formazione di un'identità autodeterminata ma percepita all'esterno come autorevole e unitaria.

La forte impronta scolastica della formazione degli operatori culturali domenicani contribuisce a ideare uno stile di traduzione che mira innanzi tutto alla chiarezza e all'univocità dell'interpretazione, bilancia, quindi, il sensum e il verbum del testo di partenza a seconda del genere testuale e della reverenza nei confronti della fonte, predispone glossa ed esegesi in modo definito, senza

57 Sul dibattito culturale si veda Epistolario di Coluccio Salutati. In generale sulla figura di Giovanni basterà qui rimandare alla voce enciclopedica Cracco, Banchini, Giovanni di Domenico e allo studio di Romano, Giovanni Dominici da Firenze.

58 Caffarini è direttore delle mantellate di Venezia e traduce la Leggenda di Vanna di Orvieto, la Leggenda di Margherita di Città di Castello, la Leggenda di Maria di Venezia (in particolare per quest'ultima si veda uno studio che vale come inquadramento anche per le altre traduzioni Sorelli, La santità imitabile. 
influenzare il contenuto del messaggio per preservarne la veritas insita, talvolta, anche nell'ordo verborum.

Lo sviluppo della traduttologia medievale manifesta le conseguenze del processo evolutivo della società: se si osserva questo fenomeno come un modello astratto, si riconosce nella partecipazione attiva dell'Ordine dei Predicatori un fattore influenzante giacché esso interpreta, e manipola, le esigenze della comunità attraverso il controllo della cultura, e modifica dunque l'andamento del modello. Pertanto, applicando la definizione di Thomas Kuhn riguardante l'epistemologia delle scienze dure, ${ }^{59}$ credo che considerare nell'analisi i contesti sociali in cui si elaborano i fenomeni culturali provochi un "cambiamento di paradigma” all'interno del sistema critico e metodologico della traduttologia medievale.

59 Khun, The Structures of Scientific Revolutions. 
